\title{
Chiral extrapolation of the charged-pion magnetic polarizability with Padé approximant
}

\author{
Fangcheng He, ${ }^{1,2}$ Derek B. Leinweber ${ }^{3}$ Anthony W. Thomas, ${ }^{3}$ and Ping Wang ${ }^{1}$ \\ ${ }^{1}$ Institute of High Energy Physics, CAS, Beijing 100049, China \\ ${ }^{2}$ CAS Key Laboratory of Theoretical Physics, Institute of Theoretical Physics, CAS, Beijing 100190, China \\ ${ }^{3}$ Centre for the Subatomic Structure of Matter (CSSM), Department of Physics, \\ University of Adelaide, Adelaide, South Australia 5005, Australia
}

\begin{abstract}
The background magnetic-field formalism of lattice QCD has been used recently to calculate the magnetic polarizability of the charged pion. These $n_{f}=2+1$ numerical simulations are electroquenched, such that the virtual sea-quarks of the QCD vacuum do not interact with the background field. To understand the impact of this, we draw on partially quenched chiral perturbation theory. In this case, the leading term proportional to $1 / M_{\pi}$ arises at tree level from $\mathcal{L}_{4}$. To describe the results from lattice QCD, while maintaining the exact leading terms of chiral perturbation theory, we introduce a Padé approximant designed to reproduce the slow variation observed in the lattice QCD results. Two-loop contributions are introduced to assess the systematic uncertainty associated with higher-order terms of the expansion. Upon extrapolation, the magnetic polarizability of the charged pion at the physical pion mass is found to be $\beta_{\pi^{ \pm}}=-1.70(14)_{\text {stat }}(25)_{\text {syst }} \times 10^{-4} \mathrm{fm}^{3}$, in good agreement with the recent experimental measurement.
\end{abstract}

\section{INTRODUCTION}

Determining the electromagnetic structure of baryons and mesons presents a contemporary challenge of broad interest in hadron physics. The internal structure of hadrons is governed by the interactions between quarks and gluons described by quantum chromodynamics (QCD). Although QCD is well established to describe the strong interactions, it is very difficult to study hadronic physics using QCD directly, due to its nonperturbative behavior.

Many phenomenological models as well as effective field theory have been utilized to learn about the mechanisms at play in determining the hadron spectrum, hadron structure and hadronic interactions. The most rigorous way to study hadron physics is through lattice QCD. It is based on the first principles of the quantum field theory and provides an avenue for the $a b$ initio calculation of Green functions via simulations of the path integral in a discrete space-time lattice.

The electromagnetic polarizability is a fundamental property characterizing the structure of a hadron. The observable reflects a dynamical response of a hadron to an external electromagnetic probe. As the lightest meson, the pion polarizability is of special interest. It is very difficult to measure accurately, due to the short lifetime of the pion $[1,2]$. A relatively recent measurement by the COMPASS collaboration [2] provides $\beta_{\pi^{ \pm}}=(-2.0 \pm 0.6 \pm 0.7) \times 10^{-4} \mathrm{fm}^{3}$ for the charged pion. Here the uncertainties are statistical and systematic respectively.

This experimental measurement is complemented by theoretical calculations based on models, such as the quark confinement model [3], Nambu-Jona-Lasinio model $[4,5]$, linear sigma model [6], dispersion sum rules [7, 8], as well as chiral perturbation theory [9, 10].

In this investigation we analyze lattice QCD results for the magnetic polarizability of the charged pion determined via the uniform background magnetic field formalism. Early work with the background-field approach calculated baryon magnetic moments $[11,12]$. The first attempt to calculate a polarizability with the background field method was made by Fiebig et al. [13]. The formalism for calculating the magnetic polarizability of a baryon within the background field method was outlined in Ref. [14].

Today, there are several calculations of light-hadron magnetic polarizabilities using the background-field formalism [15-21], with advances in algorithms complementing increased supercomputing resources to significantly reduce both systematic and statistical errors over time. In addition, first attempts to calculate the polarizability of light nuclei [22] have been made. More recently, Landau and Laplacian $S U(3) \times U(1)$ projection methods have been created to isolate the ground states of hadrons in an external magnetic field [23-25].

While the chiral extrapolation of the magnetic polarizability of the nucleon and neutral pion has been considered [23, 24, 26, 27], a chiral extrapolation of lattice QCD results for the charged-pion magnetic polarizability $\beta_{\pi \pm}$ remains.

In this paper, we will extrapolate the lattice results of Ref. [25] for $\beta_{\pi^{ \pm}}$to the physical pion mass. The results of Ref. [25] employ a new Laplacian-mode projection technique that isolates the state of interest and enables accurate determinations of the small energy shifts induced by the background magnetic field. We will analyze the one loop diagrams from partially quenched chiral perturbation theory to identify the leading contributions of quark-flow connected and disconnected diagrams separately. 


\section{PARTIALLY QUENCHED $\chi$ PT}

A naive approach to chiral extrapolation is to simply use low-order polynomial fit functions of the quark mass to fit the lattice results. However, such a procedure is not correct as it neglects the effects of the meson cloud, which can produce terms nonanalytic in the quark mass. These terms can generate rapid variation in observables for pion masses below 400 to $500 \mathrm{MeV}$ [28]. The nonanalytic terms are crucial in obtaining the correct extrapolated results at the physical pion mass.

Chiral perturbation theory $(\chi \mathrm{PT})$ provides a robust framework for determining the nonanalytic terms and their coefficients. The coefficients are model-independent and should not be taken as fit parameters. This approach has been used to extrapolate many hadronic observables [29-37].

For the magnetic polarizability of the charged pion, the tree level contribution starting from the next-to-leading order Lagrangian $\mathcal{L}_{4}$ provides a leading term to $\beta_{\pi^{ \pm}}$of order $1 / M_{\pi}$ with a well-determined coefficient [9]. The leading nonanalytic contribution to the Compton amplitude proportional to $\log \left(m_{\pi}\right)$ has its origin in the twoloop diagrams of $\chi \mathrm{PT}[10]$.

We find that the lattice QCD results for $\beta_{\pi^{ \pm}}$are described very well over the available pion-mass range by a Padé approximant involving three terms. This approximation provides an interpolation between the light quark-mass regime where $\chi \mathrm{PT}$ is robust to the larger quark-mass regime where the lattice-QCD results are smooth and slowly varying as a function of the quark mass. A similar approach was explored in Ref. [38] where baryon magnetic moments were extrapolated to the physical point.

To explore the systematic errors of the approach and the importance of higher-order terms in the chiral expansion, two-loop contributions are also considered [10]. These contributions are found to be small relative to the leading contributions. With this consideration, the magnetic polarizability of the charged pion at the physical point is $\beta_{\pi^{ \pm}}=-1.70(14)_{\text {stat }}(25)_{\text {syst }} \times 10^{-4} \mathrm{fm}^{3}$, in good agreement with the recent experimental measurement of Ref. [2].

For pion-photon scattering, the Taylor expansion of the Compton amplitude in photon energies at threshold can be expressed as

$$
\begin{aligned}
T=-2\left[\vec{\epsilon}_{1} \cdot \vec{\epsilon}_{2}^{*}\left(e^{2}-4 \pi M_{\pi} \alpha_{\pi} \omega_{1} \omega_{2}\right)-\right. \\
\left.4 \pi M_{\pi} \beta_{\pi}\left(\vec{q}_{1} \times \vec{\epsilon}_{1}\right) \cdot\left(\vec{q}_{2} \times \vec{\epsilon}_{2}^{*}\right)+\cdots\right],
\end{aligned}
$$

where $\alpha_{\pi}$ and $\beta_{\pi}$ are called the electromagnetic polariz- abilities. There have been several calculations addressing the pion electromagnetic polarizability within chiral effective field theory $[9,10,39]$. The chiral Lagrangian is organized in the following terms

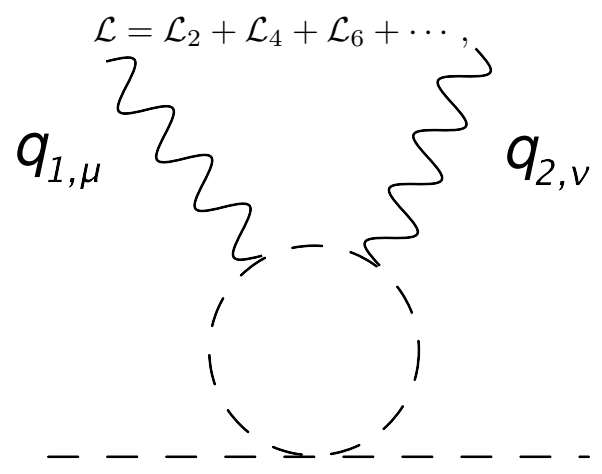

FIG. 1: The leading one-loop diagram for the pion magnetic polarizability

where the subscripts refer to the chiral order. For the one-loop diagrams, only the Lagrangian at leading order $\mathcal{L}_{2}$ is used. The expression for $\mathcal{L}_{2}$ is

$$
\mathcal{L}_{2}=\frac{f_{\pi}^{2}}{4} \operatorname{Tr}\left[D_{\mu} U D^{\mu} U^{\dagger}\right]+\frac{f_{\pi}^{2}}{4} \operatorname{Tr}\left[m\left(U+U^{\dagger}\right)\right],
$$

where $U=e^{2 i \phi / f_{\pi}}$ and $f_{\pi}=92.4(3) \mathrm{MeV}$ is the pion decay constant [40]. $\phi$ is the matrix of pseudoscalar fields

$$
\phi=\frac{1}{\sqrt{2}}\left(\begin{array}{ccc}
\frac{1}{\sqrt{2}} \pi^{0}+\frac{1}{\sqrt{6}} \eta & \pi^{+} & K^{+} \\
\pi^{-} & -\frac{1}{\sqrt{2}} \pi^{0}+\frac{1}{\sqrt{6}} \eta & K^{0} \\
K^{-} & \bar{K}^{0} & -\frac{2}{\sqrt{6}} \eta
\end{array}\right),
$$

and $m$ is the mass matrix expressed as

$$
m=\left(\begin{array}{ccc}
M_{\pi}^{2} & 0 & 0 \\
0 & M_{\pi}^{2} & 0 \\
0 & 0 & 2 M_{K}^{2}-M_{\pi}^{2}
\end{array}\right)
$$

In our extrapolation, the next higher order Lagrangian will provide the leading tree level contribution. The oneloop Feynman diagram for the magnetic polarizability of the charged pion is shown in Fig. 1.

For the charged pion, the amplitude of Fig. 1 is written as

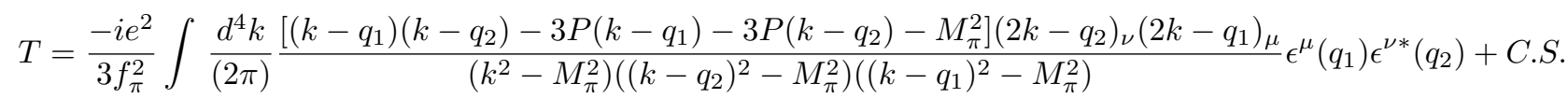



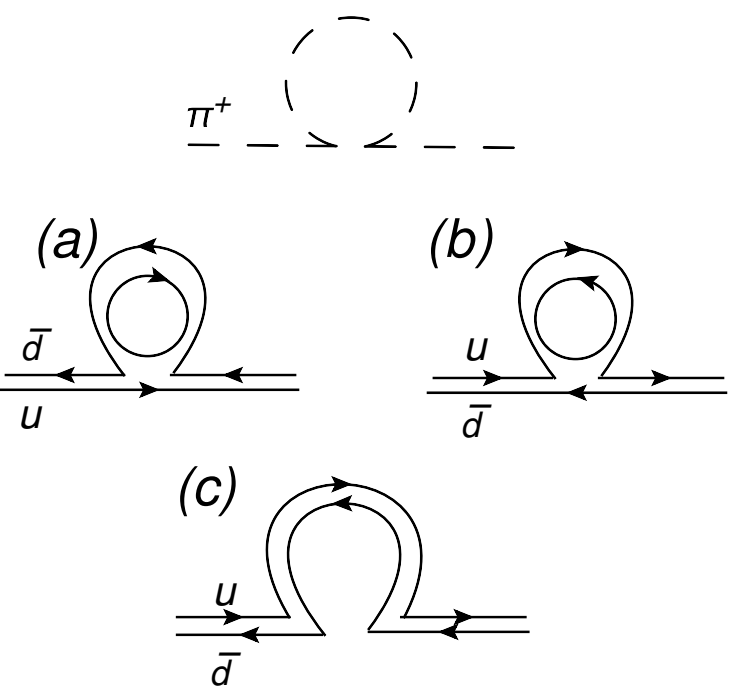

FIG. 2: Quark-flow diagrams for the $\pi^{+} \pi^{+} \pi^{+} \pi^{+}$channel. The couplings for Figs. 2(a) and 2(b) can be obtained individually by replacing the light sea-quark-loop flavor with a strange quark flavor and using SU(3)-flavor symmetry [41].

where C.S. denotes crossing symmetry terms where the photons labeled $q_{1, \mu}$ and $q_{2, \nu}$ in Fig. 1 couple with the opposite time ordering. This amplitude has no contribution to the magnetic polarizability of the charged pion.

The lattice results in [25] are simulated in the electroquenched approximation. In this case, virtual seaquark loops in the QCD vacuum do not interact with the background magnetic field. To understand the impact of this approximation, we draw on partially quenched chiral perturbation theory to separate the contributions of sea-quark loops and understand their role in composing the properties of QCD.

$\mathrm{Hu}$ et al. [42] have performed a comprehensive calculation of the pion polarizability at one loop using the graded-symmetry formalism of partial quenching for iso- lating sea-quark-loop contributions [43]. Here we complement this approach with a simple diagrammatic approach [41] where the sea-quark-loop contributions are isolated by changing the sea-quark flavor to a flavor that does not appear in the hadron under consideration, in this case a strange quark. Drawing on established SU(3) flavor relations the loop contribution is readily obtained.

The quark-flow diagrams for the $\pi^{+} \pi^{+} \pi^{+} \pi^{+}$channel are plotted in Fig. 2. The coefficients of the four-meson vertex for Figs. 2(a) and (b) alone can be obtained by replacing the sea quark with a strange quark [41] as described above. Therefore, the amplitude can be obtained by calculating a $K$-meson loop dressing with the $K$-meson mass set equal to the pion mass. The amplitude of Fig. 2(a) is written as

$T^{(a)}=\frac{-i e^{2}}{6 f_{\pi}^{2}} \int \frac{d^{4} k}{(2 \pi)} \frac{\left[\left(k-q_{1}\right)\left(k-q_{2}\right)-3 P\left(k-q_{1}\right)-3 P\left(k-q_{2}\right)-M_{\pi}^{2}\right]\left(2 k-q_{2}\right)_{\nu}\left(2 k-q_{1}\right) \mu}{\left(k^{2}-M_{\pi}^{2}\right)\left(\left(k-q_{2}\right)^{2}-M_{\pi}^{2}\right)\left(\left(k-q_{1}\right)^{2}-M_{\pi}^{2}\right)}\left(q_{1}\right) \epsilon^{\nu *}\left(q_{2}\right)+C . S .$.

Except for the leading factor, Eq. (7) is the same as Eq. (6) and once again this amplitude does not contribute to the magnetic polarizability of the charged pion. Since the expression of Fig. 2(b) has the same structure, its contribution also vanishes. Since the sum of the contributions of Figs. 2(a) and (b) matches the total amplitude of Fig. 1, the $4 \pi$ vertex in Fig. 2(c) also vanishes. These results are consistent with the conclusions from graded symmetry [43] and the comprehensive analysis of $\mathrm{Hu}$ et al. [42].

Thus, the one-loop diagrams of the leading order Lagrangian $\mathcal{L}_{2}$ make no contributions to $\beta_{\pi^{ \pm}}$in either full QCD or electroquenched QCD. Loop contributions commence at the two-loop level. This is in contrast with the $\pi^{0}$ case, where quark-annihilation contractions of quark-field operators within the neutral-pion interpolat- 
ing fields create source-sink-disconnected contributions to $\beta_{\pi^{0}}$. Similar contractions do not exist in the charged pion interpolators and thus this one-loop amplitude does not appear for the charged-pion magnetic polarizability.

The lowest order tree-level contribution starts from $\mathcal{L}_{4}$ and can be written as

$$
\beta_{0}=-\frac{\alpha}{16 f_{\pi}^{2} M_{\pi} \pi^{2}} \frac{\bar{l}_{\Delta}}{3},
$$

where $\alpha=1 / 137$ is the fine structure constant, $\bar{l}_{\Delta}=3.0 \pm$ 0.3 is the renormalized constant taken from Refs. [9, 10]. This is the leading contribution to the magnetic polarizability of the charged pion, as it is proportional to $1 / M_{\pi}$. At the physical point, this contribution is $-2.98(30) \times 10^{-4} \mathrm{fm}^{3}$.

Note, for the $\pi^{0}$ case, the leading tree-level contribution is of order $M_{\pi}$. There, sigma-meson exchange, contributing at order $1 / M_{\pi}$, was included to describe the pion mass dependence of the lattice results [27]. Here however, it is not necessary to introduce sigma exchange as the $1 / M_{\pi}$ contribution is effectively included in the $\bar{l}_{\Delta}$ term.

\section{PADÉ APPROXIMANT}

The tree level contribution from $\mathcal{L}_{6}$ is $\mathcal{O}\left(M_{\pi}\right)$. Thus the tree-level contribution up to $M_{\pi}^{3}$ can be written

$$
\beta_{\text {tree }}^{\pi^{ \pm}}=\beta_{0}+b_{1} M_{\pi}+b_{3} M_{\pi}^{3}=\beta_{0}\left(1+c_{1} M_{\pi}^{2}+c_{3} M_{\pi}^{4}\right) .
$$

To create a function able to interpolate between the light quark-mass regime where $\chi \mathrm{PT}$ is robust to the larger quark-mass regime where the lattice-QCD results are smooth and slowly varying, we consider the following Padé approximant

$$
\beta_{L}^{\pi^{ \pm}}=\beta_{0} \frac{1+c_{1} M_{\pi}^{2}+c_{3} M_{\pi}^{4}}{1+c_{4} M_{\pi}^{4}},
$$

where $c_{1}=\frac{3 a_{1}^{r}}{32 f_{\pi}^{2} \pi^{2} \bar{l}_{\Delta}}$ and $a_{1}^{r}=-3.2$ is the renormalized low-energy constant as determined in Ref. [10]. We note that the result for $\beta_{L}^{\pi^{ \pm}}$is insensitive to the value taken for $a_{1}^{r}$ as the fit parameters $c_{3}$ and $c_{4}$ compensate for changes in $a_{1}^{r}$ as the fit function is constrained by the lattice QCD results.

We note that the effectiveness of a Padé in such circumstances was illustrated by a study of the exactly soluble Euler-Heisenberg effective action, where an approximant was shown to yield a surprisingly accurate fit to the exact result provided the logarithmic behavior at small mass and the inverse power behavior at large mass was correctly incorporated [44]. The parameters $c_{3}$ and $c_{4}$ are fit parameters constrained by fitting the lattice QCD

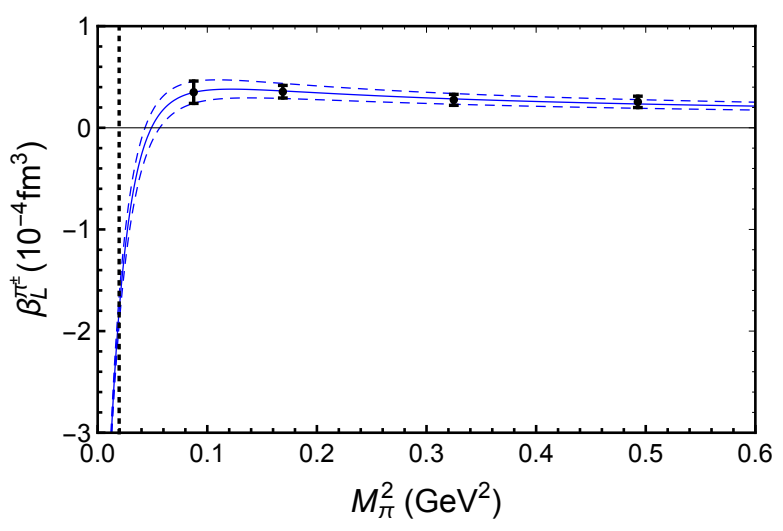

FIG. 3: Pion mass dependence of the magnetic polarizability of the charged pion. The Padé approximant of Eq. (10) (solid curve) is constrained by the CSSM lattice results of Ref. [25] (black bullets) as described in the text. The leading and next-to-leading contributions are constrained by $\chi \mathrm{PT}[9,10]$. Dashed curves represent the $1 \sigma$ error bounds associated with the statistical uncertainties of the lattice results. The vertical dotted line indicates the physical pion-mass point.

results. We find

$$
c_{3}=-0.59 \pm 0.16 \mathrm{fm}^{4}, \quad \text { and } \quad c_{4}=1.50 \pm 0.15 \mathrm{fm}^{4} .
$$

The pion mass dependence of the lattice QCD results for the charged-pion magnetic polarizability is illustrated in Fig. 3. The fit of $\beta_{L}^{\pi^{ \pm}}$to these lattice results is illustrated as a solid curve. One observes the lattice results can be described very well by the Padé approximant. The Padé approximant incorporates a heavy quark behavior consistent with the lattice QCD observations, allowing the fit function to become flat at larger pion masses, which is of course not possible with a polynomial expansion. At small pion masses, $\beta_{L}^{\pi^{ \pm}}$is dominated by the results of $\chi \mathrm{PT}$, decreasing quickly with decreasing $M_{\pi}$ due to the leading order term proportional to $1 / M_{\pi}$. As a result, the positive lattice results at large $M_{\pi}$ change to negative values at small $M_{\pi}$. At the physical pion mass, $\beta_{\pi^{ \pm}}=-1.80(14) \times 10^{-4} \mathrm{fm}^{3}$.

This extrapolation is possible because there is knowledge of $\bar{l}_{\Delta}$ from chiral perturbation theory. The merit of the extrapolation lies not only in providing a prediction to confront experiment, but also in the guidance it provides for next generation lattice QCD simulations to both directly observe the predicted sign change in the magnetic polarizability, and determine the value of $\bar{l}_{\Delta}$ from the first principles of QCD. To constrain $\bar{l}_{\Delta}$, one needs precise lattice QCD results at small pion masses. However, lattice calculations have yet to resolve a signal there. If chiral fermion actions are required to circumvent difficulties associated with additive mass renormalization issues with Wilson-clover fermions, a two-order of magnitude increase in computational resources will be required. 
To investigate the importance of higher-order terms in the chiral expansion, we proceed to include additional two-loop contributions from $\chi \mathrm{PT}[10]$. In presenting these contributions, we begin by simply adding the twoloop contributions to the existing fit illustrated in Fig. 3. The modified Padé approximant incorporating the twoloop contributions can be expressed as

$$
\beta_{F}^{\pi^{ \pm}}=\beta_{0} \frac{1-\frac{3\left(d_{1+-}-d_{1-}\right)}{32 \pi^{2} f_{\pi}^{2} \bar{l}_{\Delta}} M_{\pi}^{2}+c_{3} M_{\pi}^{4}}{1+c_{4} M_{\pi}^{4}},
$$

where $d_{1+}$ and $d_{1-}$ are coefficients for the two-loop contributions [10]. They are defined as

$$
\begin{aligned}
d_{1+} & =8 b^{r}-\frac{4}{9}\left\{l\left(l+\frac{1}{2} \bar{l}_{l}+\frac{3}{2} \bar{l}_{2}\right)-\frac{53}{24} l+\frac{1}{2} \bar{l}_{1}+\frac{3}{2} \bar{l}_{2}\right. \\
& \left.+\frac{91}{72}+\Delta_{+}\right\}, \\
d_{1-} & =a_{1}^{r}+8 b^{r}-\frac{4}{3}\left\{l\left(\bar{l}_{1}-\bar{l}_{2}+\bar{l}_{\Delta}-\frac{65}{12}\right)-\frac{1}{3} \bar{l}_{1}-\frac{1}{3} \bar{l}_{2}\right. \\
& \left.+\frac{1}{4} \bar{l}_{3}-\bar{l}_{\Delta} \bar{l}_{4}+\frac{187}{104}+\Delta_{-}\right\},
\end{aligned}
$$

with

$$
\Delta_{+}=\frac{8105}{576}-\frac{135}{64} \pi^{2}, \quad \text { and } \quad \Delta_{-}=\frac{41}{432}-\frac{53}{64} \pi^{2},
$$

where $l \equiv \ln \left(M_{\pi}^{2} / \mu^{2}\right)$, and $\bar{l}_{i}$ are scale-independent lowenergy constants (LECs) defined in Eqs. (3.8), (3.9) and (3.10) of Ref. [10].

$$
\begin{aligned}
\bar{l}_{1}=-0.4 \pm 0.6, & \bar{l}_{2}=4.3 \pm 0.1, \\
\bar{l}_{3}=2.9 \pm 2.4, & \bar{l}_{4}=4.4 \pm 0.2, \\
\bar{l}_{\Delta}=3.0 \pm 0.3 . &
\end{aligned}
$$

The scale $\mu=0.770 \mathrm{GeV}$ is set to the $\rho$-meson mass. The uncertainty in each of these LECs contributes to a systematic uncertainty in the magnetic polarizability $\beta_{\pi^{ \pm}}$. We consider the uncertainty associated with each LEC and combine their contributions in quadrature.

Our final results for the chiral extrapolation of the magnetic polarizability of the charged pion is illustrated in Fig. 4. The blue line is our previous fit of Eq. (10) to the lattice results. The red line for $0 \leq M_{\pi}^{2} \leq 2 M_{\pi}^{2 \text { Phys }}$ represents the chiral extrapolation with the two-loop contributions added to our previous fit as described by Eq. (12). The addition of the two-loop contributions makes only a small adjustment to the chiral extrapolation. At the physical point, the two-loop correction is $0.10 \times 10^{-4} \mathrm{fm}^{3}$, a $6 \%$ correction. However the correction decreases as one moves to heavier pion masses. Given that the corrections are very small and it is not clear to what extent electroquenched simulations incorporate these effects, we propose the addition of two-loop effects to provide the better estimate of the observable, and adopt the difference between the red and blue curves as contributing to the systematic error, added in quadra-

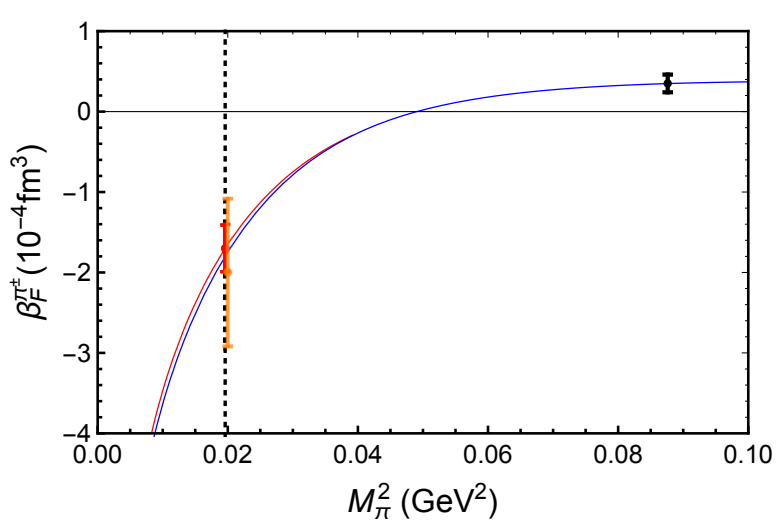

FIG. 4: Chiral extrapolation of the charged-pion magnetic polarizability. The red and blue lines illustrate the Padé approximates of Eqs. (12) and (10) with and without the two-loop contributions respectively. The full QCD prediction for the magnetic polarizability of the charged pion $\beta_{\pi \pm}$ is illustrated at the physical pion mass by the red point where the error bar incorporates both statistical and systematic uncertainties as described in the text. The experimental measurement by the COMPASS collaboration [2] is illustrated by the orange point at the physical pion mass.

ture.

The full QCD prediction for the magnetic polarizability of the charged pion $\beta_{\pi^{ \pm}}$is illustrated at the physical pion mass in Fig. 4 by the red point where the error bar incorporates both statistical and systematic uncertainties combined in quadrature. Our final estimate is $\beta_{\pi^{ \pm}}=-1.70(14)_{\text {stat }}(25)_{\text {syst }} \times 10^{-4} \mathrm{fm}^{3}$.

The experimental measurement obtained by the COMPASS collaboration [2] is $\beta_{\pi^{ \pm}}=(-2.0 \pm 0.6 \pm 0.7) \times 10^{-4}$ $\mathrm{fm}^{3}$ under the assumption $(\beta+\alpha)^{\pi}=0$. Here the uncertainties are statistical and systematic, respectively. This measurement is illustrated by the orange point in Fig. 4 where the statistical and systematic uncertainties have been added in quadrature. Our result is in good agreement with the experimental measurement.

It is interesting to examine how a power-series expansion of the Padé generates corrections to the leading contribution of $\beta_{0}$. We commence with a Taylor expansion of the total contribution without the two-loop correction, in Eq. (10)

$$
\beta_{L}^{\pi^{ \pm}}=\beta_{0}\left(1+c_{1} M_{\pi}^{2}+\left(c_{3}-c_{4}\right) M_{\pi}^{4}\right)+\cdots .
$$

Table I, lists the contributions at different orders of the expansion evaluated at the physical pion mass. Recall that the coefficient $c_{1}$ is related to $a_{1}^{r}$, known from chiral perturbation theory, whereas $c_{3}$ and $c_{4}$ are constrained by the current lattice QCD results.

We note that the large contribution at $\propto M_{\pi}^{4}$ may be a reflection of the Padé considered where a ratio of $c_{3} / c_{4}$ is encountered at large pion mass. For example, a Padé involving a ratio of $M_{\pi}^{6}$ terms may shift the strength observed at $M_{\pi}^{4}$ to neighboring terms. On the other hand, 
TABLE I: Comparison of the contributions to the charged pion magnetic polarizability in the standard units of $\times 10^{-4} \mathrm{fm}^{3}$.

\begin{tabular}{lcc}
\hline \hline Description & Term & Value $\left(\times 10^{-4} \mathrm{fm}^{3}\right)$ \\
\hline Total contribution without the two-loop correction & Eq. $(10)$ & -1.80 \\
\hline Tree level contribution at leading order & $\beta_{0}$ & -2.98 \\
Correction $\propto M_{\pi}^{2}$ & $\beta_{0} c_{1} M_{\pi}^{2}$ & 0.07 \\
Correction $\propto M_{\pi}^{4}$ & $\beta_{0}\left(c_{3}-c_{4}\right) M_{\pi}^{4}$ & 1.60 \\
Sum of remaining contributions & $\beta_{L}^{\pi^{ \pm}}-\beta_{0}\left(1+c_{1} M_{\pi}^{2}+\left(c_{3}-c_{4}\right) M_{\pi}^{4}\right)$ & -0.49 \\
\hline Two loop correction of Eq. (12) & $-\frac{3\left(d_{1+}-d_{1-}+a_{1}^{r}\right)}{32 \pi^{2} f_{\pi}^{2} \bar{l}_{\Delta}\left(1+c_{4} M_{\pi}^{4}\right)} M_{\pi}$ & 0.10 \\
\hline Full QCD prediction & $\mathrm{Eq} .(12)$ & -1.70 \\
\hline \hline
\end{tabular}

these coefficients are required to describe the lattice QCD results. Therefore, the large contribution at $M_{\pi}^{4}$ cautions against the naive application of the power-series expansion, even at the physical pion mass.

Considering the corrections $\propto M_{\pi}^{4}$ and higher order contributions together, we observe an $\mathcal{O}\left(M_{\pi}^{4}\right)$ correction of $1.11 \times 10^{-4} \mathrm{fm}^{3}$ relative to a leading order term of magnitude $2.98 \times 10^{-4} \mathrm{fm}^{3}$, a $37 \%$ correction at the physical pion mass.

\section{SUMMARY}

In this paper, we have investigated the magnetic polarizability of the charged pion based on an analysis of recent lattice QCD simulations at a range of quark masses. We considered partially-quenched chiral perturbation theory to understand the role of sea-quark-loops in the magnetic polarizability of the charged pion at oneloop level, vital to understanding the impact of electroquenching in the lattice QCD simulations. In this case, electroquenched and full QCD agree, with neither theory making contributions to the charged-pion magnetic polarizability at one-loop level. Thus the fact that the lattice simulations are electroquenched has no impact on the leading loop contributions to the magnetic polarizability.

To interpolate between the light quark-mass regime where $\chi \mathrm{PT}$ is robust to the larger quark-mass regime where the lattice-QCD results are smooth and slowly varying, a Padé approximant was constructed. The lattice results are described well by the Padé approximant. To evaluate the impact of higher-order contributions in the chiral extrapolation, two-loop contributions as determined by Ref. [10] were investigated. The contribution at the physical pion mass is small and decreases as one moves to larger pion masses.

Although the lattice results at larger pion masses are positive, the final result at the physical pion mass is negative at $\beta_{\pi^{ \pm}}=-1.70(14)_{\text {stat }}(25)_{\text {syst }} \times 10^{-4} \mathrm{fm}^{3}$, in very good agreement with the experimental measurement by the COMPASS collaboration [2].

Future research will focus on studying the pion mag- netic polarizability at smaller pion masses, to directly observe the sign change of $\beta_{\pi^{ \pm}}$predicted in this analysis. Such simulations are very demanding, particularly if the large statistical fluctuations observed at light quark masses are associated with additive-mass renormalization issues in nonchiral fermion actions. If one needs a chiral fermion action to circumvent this problem, a two-order of magnitude in computational resources will be required.

Understanding the finite volume corrections to the charged pion magnetic polarizability remains of interest, and can be quantified through simulations on larger volumes.

An alternative approach to the background field formalism is to access the polarizability via perturbative electromagnetic-current insertions in four-point correlation function calculations [45, 46]. Here the QCD basis states are not mixed by the electromagnetic interactions and may be of advantage in understanding $\Sigma^{0}$ and $\Lambda$ polarizabilities for example, as these states mix in the background-field formalism.

Finally, while the two-loop contributions are remarkably small, in principle it would be interesting to bring the graded-symmetry approach of partially-quenched chiral perturbation theory to the two-loop $\chi \mathrm{PT}$ calculations to learn the details of electroquenching in the two-loop sector.

\section{Acknowledgement}

This research was supported with supercomputing resources provided by the Phoenix HPC service at the University of Adelaide. This research was undertaken with the assistance of resources from the National Computational Infrastructure (NCI), provided through the National Computational Merit Allocation Scheme, and supported by the Australian Government through Grants No. LE190100021, LE160100051 and the University of Adelaide Partner Share. This research was supported by the Australian Research Council through ARC Discovery Project Grants No. DP180100497 (A.W.T) and DP150103164, DP190102215 and DP210103706 (D.B.L), and by the National Natural Sciences Foundations of China under the grant No. 11975241. 
[1] J. Ahrens et al. Eur. Phys. J. A23 (2005) 113-127, arXiv:nucl-ex/0407011 [nucl-ex].

[2] COMPASS Collaboration, C. Adolph et al. Phys. Rev. Lett. 114 (2015) 062002, arXiv:1405.6377 [hep-ex].

[3] M. A. Ivanov and T. Mizutani Phys. Rev. D45 (1992) $1580-1601$.

[4] V. Bernard and D. Vautherin Phys. Rev. D40 (1989) 1615.

[5] A. E. Dorokhov, M. K. Volkov, J. Hufner, S. P. Klevansky, and P. Rehberg Z. Phys. C75 (1997) 127-135.

[6] V. Bernard, B. Hiller, and W. Weise Phys. Lett. B205 (1988) 16-21.

[7] L. V. Filkov, I. Guiasu, and E. E. Radescu Phys. Rev. D26 (1982) 3146.

[8] J. F. Donoghue and B. R. Holstein Phys. Rev. D48 (1993) 137-146, arXiv:hep-ph/9302203 [hep-ph] .

[9] U. Burgi Nucl. Phys. B479 (1996) 392-426, arXiv:hep-ph/9602429 [hep-ph].

[10] J. Gasser, M. A. Ivanov, and M. E. Sainio Nucl. Phys. B745 (2006) 84-108, arXiv:hep-ph/0602234 [hep-ph].

[11] G. Martinelli, G. Parisi, R. Petronzio, and F. Rapuano Phys. Lett. B 116 (1982) 434-436.

[12] C. W. Bernard, T. Draper, K. Olynyk, and M. Rushton Phys. Rev. Lett. 49 (1982) 1076.

[13] H. R. Fiebig, W. Wilcox, and R. M. Woloshyn Nucl. Phys. B 324 (1989) 47-66.

[14] M. Burkardt, D. B. Leinweber, and X.-m. Jin Phys. Lett. B385 (1996) 52-56, arXiv:hep-ph/9604450 [hep-ph].

[15] F. X. Lee, L. Zhou, W. Wilcox, and J. C. Christensen Phys. Rev. D 73 (2006) 034503, arXiv:hep-lat/0509065.

[16] T. Primer, W. Kamleh, D. Leinweber, and M. Burkardt Phys. Rev. D 89 no. 3, (2014) 034508, arXiv:1307.1509 [hep-lat].

[17] E. Luschevskaya, O. Solovjeva, O. Kochetkov, and O. Teryaev Nucl. Phys. B 898 (2015) 627-643, arXiv:1411.4284 [hep-lat].

[18] E. Luschevskaya, O. Solovjeva, and O. Teryaev Phys. Lett. B $\mathbf{7 6 1}$ (2016) 393-398, arXiv:1511.09316 [hep-lat].

[19] G. S. Bali, B. B. Brandt, G. Endrődi, and B. Gläßle Phys. Rev. D97 no. 3, (2018) 034505, arXiv:1707.05600 [hep-lat].

[20] R. Bignell, W. Kamleh, and D. Leinweber Phys. Rev. D100 no. 11, (2020) 114518, arXiv:1910.14244 [hep-lat]. [Phys. Rev.D100,114518(2019)].

[21] H.-T. Ding, S.-T. Li, A. Tomiya, X.-D. Wang, and Y. Zhang arXiv:2008.00493 [hep-lat].

[22] NPLQCD Collaboration, E. Chang, W. Detmold, K. Orginos, A. Parreno, M. J. Savage, B. C. Tiburzi, and S. R. Beane Phys. Rev. D 92 no. 11, (2015) 114502, arXiv:1506.05518 [hep-lat].

[23] R. Bignell, J. Hall, W. Kamleh, D. Leinweber, and M. Burkardt Phys. Rev. D98 no. 3, (2018) 034504, arXiv:1804.06574 [hep-lat].

[24] R. Bignell, W. Kamleh, and D. Leinweber Phys. Rev. D101 no. 9, (2020) 094502, arXiv:2002.07915 [hep-lat].
[25] R. Bignell, W. Kamleh, and D. Leinweber Phys. Lett. B811 (2020) 135853, arXiv:2005.10453 [hep-lat]

[26] J. M. M. Hall, D. B. Leinweber, and R. D. Young Phys. Rev. D89 no. 5, (2014) 054511, arXiv:1312.5781 [hep-lat].

[27] F. He, D. B. Leinweber, A. W. Thomas, and P. Wang Phys. Rev. D102 no. 11, (2020) 114509, arXiv:2010.01580 [nucl-th].

[28] A. W. Thomas Nucl. Phys. Proc. Suppl. 119 (2003) 50-58, arXiv:hep-lat/0208023 [hep-lat].

[29] D. B. Leinweber, A. W. Thomas, and R. D. Young Phys. Rev. Lett. 92 (2004) 242002, arXiv:hep-lat/0302020 [hep-lat]

[30] D. B. Leinweber, A. W. Thomas, and R. D. Young Nucl. Phys. A755 (2005) 59-70, arXiv:hep-lat/0501028 [hep-lat].

[31] C. Allton, W. Armour, D. B. Leinweber, A. W. Thomas, and R. D. Young Phys. Lett. B 628 (2005) 125-130, arXiv:hep-lat/0504022.

[32] W. Armour, C. Allton, D. B. Leinweber, A. W. Thomas, and R. D. Young J. Phys. G 32 (2006) 971-992, arXiv:hep-lat/0510078.

[33] R. D. Young, D. B. Leinweber, and A. W. Thomas Phys. Rev. D71 (2005) 014001, arXiv:hep-lat/0406001 [hep-lat].

[34] D. B. Leinweber, S. Boinepalli, I. C. Cloet, A. W. Thomas, A. G. Williams, R. D. Young, J. M. Zanotti, and J. B. Zhang Phys. Rev. Lett. 94 (2005) 212001, arXiv:hep-lat/0406002 [hep-lat].

[35] P. Wang, D. Leinweber, A. Thomas, and R. Young Phys. Rev. C 79 (2009) 065202, arXiv:0807.0944 [hep-ph].

[36] D. B. Leinweber, S. Boinepalli, A. W. Thomas, P. Wang, A. G. Williams, R. D. Young, J. M. Zanotti and J. B. Zhang Phys. Rev. Lett. 97 (2006) 022001, arXiv:hep-lat/0601025 [hep-lat].

[37] J. M. M. Hall, D. B. Leinweber, and R. D. Young Phys. Rev. D88 no. 1, (2013) 014504, arXiv:1305.3984 [hep-lat].

[38] E. J. Hackett-Jones, D. B. Leinweber, and A. W. Thomas Phys. Lett. B489 (2000) 143-147, arXiv:hep-lat/0004006 [hep-lat].

[39] J. Bijnens and F. Cornet Nucl. Phys. B296 (1988) 557-568.

[40] Particle Data Group Collaboration, D. E. Groom et al. Eur. Phys. J. C15 (2000) 1-878.

[41] D. B. Leinweber Phys. Rev. D69 (2004) 014005, arXiv:hep-lat/0211017 [hep-lat]

[42] J. Hu, F.-J. Jiang, and B. C. Tiburzi Phys. Rev. D77 (2008) 014502, arXiv:0709.1955 [hep-lat].

[43] C. W. Bernard and M. F. L. Golterman Phys. Rev. D46 (1992) 853-857, arXiv:hep-lat/9204007 [hep-lat].

[44] G. V. Dunne, A. W. Thomas, and S. V. Wright Phys. Lett. B 531 (2002) 77-82, arXiv:hep-th/0110155.

[45] W. Wilcox Annals Phys. 255 (1997) 60-74, arXiv:hep-lat/9606019.

[46] W. Wilcox and F. X. Lee arXiv:2106.02557 [hep-lat]. 